SAGA-HE-55

KYUSHU-HET-15

January 29, 1994

\title{
Fermion Scattering off CP-Violating Electroweak Bubble Wall
}

\author{
Koichi Funakubo $^{1) \# a}$, Akira Kakuto2) ${ }^{\# b}$, Shoichiro Otsuki ${ }^{2)}$, \\ Kazunori Takenaga $^{3)} \# c$ and Fumihiko Toyoda ${ }^{2)}{ }^{\# d}$ \\ 1) Department of Physics, Saga University, Saga 840 Japan \\ 2) Department of Liberal Arts, Kinki University in Kyushu, Iizuka 820 Japan \\ 3) Department of Physics, Kyushu University, Fukuoka 812 Japan
}

\begin{abstract}
A general prescription to solve the Dirac equation in the presence of CPviolating electroweak bubble wall is presented. The profile of the bubble wall is not specified except that the wall height is $m_{0}$ and zero deep in the broken- and the symmetric-phase regions, respectively, where $m_{0}$ is a fermion mass given by the Higgs-vacuum-expectation value and the Yukawa coupling. The CP-violating effects are evaluated by regarding CP-violating part of the bubble wall as a perturbation to CP-conserving solutions. The basic quantity, $R_{R \rightarrow L}-\bar{R}_{R \rightarrow L}$, which would contribute to the cosmological baryon asymmetry, is estimated for some typical profiles of the wall, where $R_{R \rightarrow L}\left(\bar{R}_{R \rightarrow L}\right)$ is the reflection coefficient of right-handed chiral fermion (anti-fermion).
\end{abstract}

\footnotetext{
\#a e-mail: funakubo@sagagw.cc.saga-u.ac.jp \#b e-mail: kakuto@fuk.kindai.ac.jp

\#c e-mail: f77498a@kyu-cc.cc.kyushu-u.ac.jp

\#d e-mail: ftoyoda@fuk.kindai.ac.jp
} 


\section{Introduction}

It is one of the most challenging problems in particle physics and cosmology to explain the baryon asymmetry of the universe. In order to generate the baryon asymmetry, the basic theory should at least satisfy the famous three conditions ${ }^{[1]}$ : there exist (i) baryon-number violation, (ii) CP nonconservation and (iii) out-ofequilibrium processes. It has been recognized that the baryon number is violated in the standard model through change of gauge-field configurations due to the axial anomaly ${ }^{[2]}$. At very high temperature, the baryon number is badly broken even to erase any primordial asymmetry, while the transition rate becomes negligible at low temperature. Thus there is a potential possibility in the standard model to produce the baryon asymmetry if the electroweak phase transition at a temperature $T \sim 100 \mathrm{GeV}$ is of first order, which guarantees the above condition (iii), and if CPnonconserving interactions are effective. It is known that the phase transition in the standard model may be weakly of first order for light Higgs boson. However, the experimental lower bound on the Higgs-boson mass $(\sim 60 \mathrm{GeV})$ seems to contradict the requirement to forbid anomalous processes induced by the sphaleron after the electroweak phase transition ${ }^{[3]}$. Furthermore it seems that the CP violation in the Kobayashi-Maskawa scheme ${ }^{[4]}$ is too feeble to generate the observed baryon asymmetry.

A simple generalization of the standard model would be to introduce another Higgs doublet ${ }^{[5]}$. In two-Higgs-doublet models, including the supersymmetric extension of the standard model, there could appear a new $\mathrm{CP}$-violating phase in the Higgs sector. It is expected that the new CP violation could make baryon asymmetry large enough to be comparable with the observed value. Moreover it is argued that the constraint on the mass of the lightest Higgs boson to avoid washing out of the baryon asymmetry after the electroweak phase transition is not so stringent that the Higgs boson could be as heavy as about $100 \mathrm{GeV}^{[5]}$. It is quite possible that the phase transition may be of first order because of many degrees of freedom in the Higgs sector ${ }^{[5]}$. In a previous paper, three of the authors ( K. F., A. K. and 
K. T. ) have shown that the electroweak phase transition is clearly of first order in a two-Higgs-doublet model with a constraint that the Higgs potential contains no quadratic terms in the symmetric phase ${ }^{[6]}$.

In this paper, we study fermion propagation in CP-violating bubble-wall background, which would play an important role to generate the cosmological baryon asymmetry $^{[7]}$. We give a general prescription to solve the Dirac equation in the presence of CP-violating bubble wall. For simplicity, we take a single species of fermion, e.g., the top quark. If the electroweak phase transition is actually of first order, supercooling will occur and bubbles of the broken phase will be generated in false vacuum. The bubbles will expand with some velocity and the space will be filled with the true vacuum. Since the bubble scale is macroscopic, the profile function of the bubble wall may be assumed to depend on a single spatial coordinate in the rest frame of the bubble wall. Far from the wall, deep in the broken-phase region, the Higgs field develops a constant vacuum expectation value. We assign a complex mass to the fermion as a function of $z, m(z)=m_{R}(z)+i m_{I}(z)$, where $z$ is the coordinate perpendicular to the wall. The real part $m_{R}(z)$ asymptotically behaves such that $m_{R}(z) \rightarrow 0$ as $z \rightarrow-\infty$ ( symmetric phase ) and $m_{R}(z) \rightarrow m_{0}$ as $z \rightarrow+\infty$ ( broken phase), where $m_{0}$ is the fermion mass. The imaginary part $m_{I}(z)$ gives rise to $\mathrm{CP}$ violation if $m_{I}(z) / m_{R}(z)$ is not a constant.

We do not specify any explicit form of the profile function $m(z)$ and obtain general expressions for the reflection and the transmission coefficients. The CPviolating effects are evaluated by regarding the $\mathrm{CP}$-violating term as a perturbation to CP-conserving solutions. Namely, we first solve the CP-conserving Dirac equation. Subsequently we solve the full equation to the first order of the CP-violating term by means of the Green's function ${ }^{[9]}$. Our method corresponds to the distorted wave Born approximation ( DWBA ). The reflection coefficient $R$ and the transmission coefficient $T$ for the fermion incident from the symmetric phase region to the wall satisfy the unitarity $R+T=1$ to the first order of perturbation theory. The

$\star$ For a special bubble-wall profile of the kink type with no CP violation, the Dirac equation has been studied by Ayala et al. ${ }^{[8]}$. 
basic quantity, $\Delta R \equiv R_{R \rightarrow L}-\bar{R}_{R \rightarrow L}$, which would be important to explain the cosmological baryon asymmetry ${ }^{[7]}$, is estimated for some typical profiles of the wall, where $R_{R \rightarrow L}\left(\bar{R}_{R \rightarrow L}\right)$ is the reflection coefficient for right-handed chiral fermion (anti-fermion). The absolute value of $\Delta R$ is found to decrease rapidly with the in-

crease of $E^{*} \equiv \sqrt{E^{2}-\boldsymbol{p}_{T}^{2}}$, where $E$ and $\boldsymbol{p}_{T}$ are energy and transverse momentum of the incident fermion, respectively. It seems that $|\Delta R|$ decreases more rapidly with the increase of $E^{*}$ for thicker walls. We expect that our prescription would serve to construct new models to generate the cosmological baryon asymmetry in the electroweak phase transition of first order.

In the next section, we will present a general prescription to solve the Dirac equation in the background of $\mathrm{CP}$-violating bubble wall. The reflection and the transmission coefficients are obtained from the solutions. In Section 3, the results in Section 2 are applied to the special case where the real part of the bubble-wall profile is of the kink type. ${ }^{[8]}$ In this case we estimate $\Delta R$ numerically by assuming typical forms for the imaginary part of the bubble-wall profile. The final section is devoted to conclusions. Details of calculations will be given in Appendix.

\section{DWBA to CP-Violating Dirac Equation}

\subsection{Dirac EQUation AND Ansatz}

Here we solve the Dirac equation in the bubble-wall background with CP violation. For simplicity, we consider one-flavor model described by the lagrangian,

$$
\mathcal{L}=\bar{\psi}_{L} i \not \psi_{L}+\bar{\psi}_{R} i \not \partial \psi_{R}+\left(f \bar{\psi}_{L} \psi_{R} \phi+\text { h.c. }\right) .
$$

In the vacuum, near the first-order phase transition point, $\langle\phi\rangle$ may be $x$-dependent field, so that we put

$$
m(\boldsymbol{x})=-f\langle\phi\rangle(\boldsymbol{x}),
$$

where $m(\boldsymbol{x})$ is in general complex-valued and we neglect the time dependence. 
If the phase of $m(\boldsymbol{x})$ has no spatial dependence, it is removed by a constant biunitary transformation. We are interested in $\boldsymbol{x}$-dependent phase which cannot be transformed away. The Dirac equation to be solved is

$$
i \not \partial \psi(t, \boldsymbol{x})-m(\boldsymbol{x}) P_{R} \psi(t, \boldsymbol{x})-m^{*}(\boldsymbol{x}) P_{L} \psi(t, \boldsymbol{x})=0
$$

where $P_{R}=\left(1+\gamma_{5}\right) / 2$ and $P_{L}=\left(1-\gamma_{5}\right) / 2$. In the bubble-wall background whose radius is sufficiently large, $m(\boldsymbol{x})$ can be regarded as a function of only one spatial coordinate, so that we put $m(\boldsymbol{x})=m(z)$.

To solve (2.3), we take the following Ansatz:

$$
\begin{aligned}
\psi(t, \boldsymbol{x}) & =\left(i \not \partial+m^{*}(z) P_{R}+m(z) P_{L}\right) \mathrm{e}^{i \sigma\left(-E t+\boldsymbol{p}_{T} \boldsymbol{x}_{T}\right)} \psi_{E}\left(\boldsymbol{p}_{T}, z\right) \\
& =\mathrm{e}^{i \sigma\left(-E t+\boldsymbol{p}_{T} \boldsymbol{x}_{T}\right)}\left[\sigma\left(\gamma^{0} E-\gamma_{T} p_{T}\right)+i \gamma^{3} \partial_{z}+m^{*}(z) P_{R}+m(z) P_{L}\right] \psi_{E}\left(\boldsymbol{p}_{T}, z\right),
\end{aligned}
$$

where $\sigma=+(-)$ for positive(negative)-energy solution, $\boldsymbol{p}_{T}=\left(p^{1}, p^{2}\right), \boldsymbol{x}_{T}=$ $\left(x^{1}, x^{2}\right), p_{T}=\left|\boldsymbol{p}_{T}\right|$ and $\gamma_{T} p_{T}=\gamma^{1} p^{1}+\gamma^{2} p^{2}$. If we put $E=E^{*} \cosh \eta$ and $p_{T}=E^{*} \sinh \eta$ with $E^{*}=\sqrt{E^{2}-p_{T}^{2}}$, the Lorentz transformation of the spinor, represented by $\psi^{\prime}=\mathrm{e}^{\eta \gamma^{0} \gamma_{T} / 2} \psi$, eliminates $\boldsymbol{p}_{T}$ from (2.4) and from the Dirac equation. Suppose that we do this Lorentz rotation for a fixed $\boldsymbol{p}_{T}$. Then the Dirac equation is rewritten, in the second-order form, as

$$
\left[E^{* 2}+\partial_{z}^{2}-|m(z)|^{2}+i m_{R}^{\prime}(z) \gamma^{3}-m_{I}^{\prime}(z) \gamma_{5} \gamma^{3}\right] \psi_{E}(z)=0
$$

where $m(z)=m_{R}(z)+i m_{I}(z)$, with both $m_{R}(z)$ and $m_{I}(z)$ being real-valued. Now let us introduce a set of dimensionless variables, using a parameter, $a$, which has a dimension of mass and whose inverse characterizes the thickness of the wall. Define

$$
\begin{aligned}
& m_{R}(z)=m_{0} f(a z)=m_{0} f(x), \\
& m_{I}(z)=m_{0} g(a z)=m_{0} g(x), \\
& x \equiv a z, \quad \tau \equiv a t, \quad \epsilon \equiv E^{*} / a, \quad \xi \equiv m_{0} / a
\end{aligned}
$$

where $m_{0}$ is the fermion mass in the broken phase. Then the Ansatz (2.4) and the 
second-order equation (2.5) are expressed as

$$
\psi(\tau, x)=\mathrm{e}^{-i \sigma \epsilon \tau}\left[\sigma \epsilon \gamma^{0}+i \gamma^{3} \frac{d}{d x}+\xi f(x)-i \xi g(x) \gamma_{5}\right] \psi_{\epsilon}(x)
$$

and

$$
\left[\epsilon^{2}+\frac{d^{2}}{d x^{2}}-\xi^{2}\left(f(x)^{2}+g(x)^{2}\right)+i \xi f^{\prime}(x) \gamma^{3}-\xi g^{\prime}(x) \gamma_{5} \gamma^{3}\right] \psi_{\epsilon}(x)=0
$$

respectively.

Our aim is to solve (2.8) and put it back into the Ansatz (2.7) to obtain the Dirac spinor, and to compute various currents from which the reflection and transmission coefficients are defined. As for $f(x)$ and $g(x)$, we do not specify their functional forms but only assume that

$$
f(x) \rightarrow \begin{cases}1, & \text { as } x \rightarrow+\infty \\ 0, & \text { as } x \rightarrow-\infty\end{cases}
$$

and that $|g(x)|<<1$, which means that the CP violation is small. (2.9) means that the system is in the broken phase at $x \sim+\infty$ while in the symmetric phase at $x \sim-\infty$.

\subsection{Outline of the DWBA to the Dirac equation}

Let us explain our strategy to construct the spinor solution. Since $|g(x)|$ is very small, we regard it as a perturbation and keep only quantities of $O\left(g^{1}\right)$. First, put

$$
\psi_{\epsilon}(x)=\psi^{(0)}(x)+\psi^{(1)}(x)
$$

where $\psi^{(0)}(x)$ is a solution to the unperturbed equation

$$
\left[\epsilon^{2}+\frac{d^{2}}{d x^{2}}-\xi^{2} f(x)^{2}+i \xi f^{\prime}(x) \gamma^{3}\right] \psi^{(0)}(x)=0
$$


with an appropriate boundary condition. Then $\psi^{(1)}(x)$ can be solved as

$$
\psi^{(1)}(x)=\int d x^{\prime} G\left(x, x^{\prime}\right) V\left(x^{\prime}\right) \psi^{(0)}\left(x^{\prime}\right)
$$

where

$$
V(x)=-\xi g^{\prime}(x) \gamma_{5} \gamma^{3}
$$

and $G\left(x, x^{\prime}\right)$ is the Green's function satisfying

$$
\left[\epsilon^{2}+\frac{d^{2}}{d x^{2}}-\xi^{2} f(x)^{2}+i \xi f^{\prime}(x) \gamma^{3}\right]_{\alpha \gamma} G_{\gamma \beta}\left(x, x^{\prime}\right)=-\delta_{\alpha \beta} \delta\left(x-x^{\prime}\right)
$$

with the same boundary condition as $\psi^{(0)}(x)$. Thus, to this order, the spinor solution to the Dirac equation is given by

$$
\psi(x) \simeq \mathrm{e}^{-i \sigma \epsilon \tau}\left\{\left[\sigma \epsilon \gamma^{0}+i \gamma^{3} \frac{d}{d x}+\xi f(x)\right]\left[\psi^{(0)}(x)+\psi^{(1)}(x)\right]-i \xi g(x) \gamma_{5} \psi^{(0)}(x)\right\} .
$$

\subsection{Solution to the UnPERTURBed EQUATion}

In the following, any explicit forms of $f(x)$ and $g(x)$ are not necessary, except for the condition (2.9). We shall present general results on the wave function and currents below. The details of the calculation are given in Appendix.

If we expand $\psi^{(0)}(x)$ in terms of the eigenspinors of $\gamma^{3}$ as $\psi^{(0)}(x) \sim \phi_{ \pm}(x) u_{ \pm}^{s}$, with

$$
\gamma^{3} u_{ \pm}^{s}= \pm i u_{ \pm}^{s} \quad(s=1,2)
$$

$\phi_{ \pm}(x)$ must satisfy

$$
\left[\epsilon^{2}+\frac{d^{2}}{d x^{2}}-\xi^{2} f(x)^{2} \mp \xi f^{\prime}(x)\right] \phi_{ \pm}(x)=0 .
$$

Because of (2.9), the asymptotic forms of $\phi_{ \pm}(x)$ should be

$$
\phi_{ \pm}(x) \rightarrow \begin{cases}\mathrm{e}^{\alpha x}, \mathrm{e}^{-\alpha x} & (x \rightarrow+\infty) \\ \mathrm{e}^{\beta x}, \mathrm{e}^{-\beta x} & (x \rightarrow-\infty)\end{cases}
$$

where $\alpha=i \sqrt{\epsilon^{2}-\xi^{2}}$ and $\beta=i \epsilon$. Here we consider the case where the scattering 
states exist at $x=+\infty ; \epsilon>\xi$. We denote the independent solutions to (2.17) as $\phi_{ \pm}^{(+\alpha)}(x)$ and $\phi_{ \pm}^{(-\alpha)}(x)=\left(\phi_{ \pm}^{(+\alpha)}(x)\right)^{*}$ which satisfy

$$
\begin{aligned}
& \phi_{ \pm}^{(+\alpha)}(x) \rightarrow \mathrm{e}^{\alpha x}, \\
& \phi_{ \pm}^{(-\alpha)}(x) \rightarrow \mathrm{e}^{-\alpha x},
\end{aligned}
$$

at $x \rightarrow+\infty$. Their asymptotic forms at $x \rightarrow-\infty$ are

$$
\begin{aligned}
& \phi_{ \pm}^{(+\alpha)}(x) \sim \gamma_{ \pm}(\alpha, \beta) \mathrm{e}^{\beta x}+\gamma_{ \pm}(\alpha,-\beta) \mathrm{e}^{-\beta x} \\
& \phi_{ \pm}^{(-\alpha)}(x) \sim \gamma_{ \pm}(-\alpha, \beta) \mathrm{e}^{\beta x}+\gamma_{ \pm}(-\alpha,-\beta) \mathrm{e}^{-\beta x}
\end{aligned}
$$

because of the symmetry of (2.17) under $\beta \mapsto-\beta$. Needless to say, $\gamma_{ \pm}(\alpha, \beta)^{*}=$ $\gamma_{ \pm}(-\alpha,-\beta)$ and $\gamma_{ \pm}(\alpha,-\beta)^{*}=\gamma_{ \pm}(-\alpha, \beta)$. With these solutions, the general solution to (2.11) can be constructed as

$$
\psi^{(0)}(x)=\sum_{s=1,2} \sum_{ \pm}\left[\left(A_{ \pm}^{s}\right)^{(-\alpha)} \phi_{ \pm}^{(-\alpha)}(x)+\left(A_{ \pm}^{s}\right)^{(+\alpha)} \phi_{ \pm}^{(+\alpha)}(x)\right] u_{ \pm}^{s}
$$

Multiplied by the operator, $\sigma \epsilon \gamma^{0}+i \gamma^{3} \frac{d}{d x}+\xi f(x)$, it becomes the general solution to the unperturbed (CP-conserving) Dirac equation,

$$
\left[\sigma \epsilon \gamma^{0}+i \gamma^{3} \frac{d}{d x}-\xi f(x)\right] \psi_{0}(x)=0
$$

As shown in Appendix, the coefficients, $\left(A_{-}^{s}\right)^{( \pm \alpha)}$, are not needed to construct the general $\psi_{0}(x)$. Eventually, the general solution to $(2.20)$ is given by

$$
\begin{aligned}
\psi_{0}(x)= & {\left[\sigma \epsilon \gamma^{0}+i \gamma^{3} \frac{d}{d x}+\xi f(x)\right] \psi^{(0)}(x) } \\
= & \sum_{s}\left\{A_{s}^{(-)}\left[\sigma \epsilon \phi_{+}^{(-\alpha)}(x) u_{-}^{s}+(\xi+\alpha) \phi_{-}^{(-\alpha)}(x) u_{+}^{s}\right]\right. \\
& \left.\quad+A_{s}^{(+)}\left[\sigma \epsilon \phi_{+}^{(+\alpha)}(x) u_{-}^{s}+(\xi-\alpha) \phi_{-}^{(+\alpha)}(x) u_{+}^{s}\right]\right\}
\end{aligned}
$$

where

$$
\psi^{(0)}(x)=\sum_{s}\left[A_{s}^{(-)} \phi_{+}^{(-\alpha)}(x)+A_{s}^{(+)} \phi_{+}^{(+\alpha)}(x)\right] u_{+}^{s}
$$

This $\psi^{(0)}(x)$ is the starting point of the DWBA. Any boundary condition can be implemented by choosing $A_{s}^{(-\alpha)}$ and $A_{s}^{(+\alpha)}$ appropriately. 
Now we impose a boundary condition to specify the scattering state. We consider a state in which the incident wave coming from $x=-\infty$ (symmetric phase) is reflected in part at the bubble wall, while at $x=+\infty$ only the transmitted wave exists. This situation is achieved by setting $A_{s}^{(-\alpha)}=0$ for $\sigma=+$ and $A_{s}^{(+\alpha)}=0$ for $\sigma=-$, respectively, in (2.21) and (2.22). The Green's function (2.14) which matches this boundary condition can be explicitly constructed from the functions $\phi_{ \pm}^{(+\alpha)}(x)$ and $\phi_{ \pm}^{(-\alpha)}(x)$, as shown in Appendix. Thus we are ready to compute the currents in the asymptotic regions.

\subsection{WAVE FUNCTION AND CURRENTS IN THE ASYMPTOTIC REGIONS}

According to (2.15), the perturbed wave can be obtained once the unperturbed wave functions and the Green's function are known. We present only the results, while the derivations are given in Appendix. The asymptotic forms of the wave function are

$$
\begin{aligned}
& \left(\psi(x)_{\sigma=+}\right)^{\text {trans }} \\
= & \mathrm{e}^{-i \epsilon \tau+\alpha x} \sum_{s} A_{s}^{(+)}\left\{(\xi-\alpha)\left[1+(-)^{s} \frac{\xi}{\epsilon}\left(\frac{\xi+\alpha}{2 \alpha} I_{1}+\frac{1}{2} g(-\infty)\right)\right] u_{+}^{s}\right. \\
& \left.+\epsilon\left[1+(-)^{s} \frac{\xi}{\epsilon}\left(\frac{\xi+\alpha}{2 \alpha} I_{1}-g(+\infty)+\frac{1}{2} g(-\infty)\right)\right] u_{-}^{s}\right\}, \\
& \left(\psi(x)_{\sigma=+}\right)^{i n c} \\
= & \mathrm{e}^{-i \epsilon \tau+\beta x} \gamma_{+}(\alpha, \beta) \sum_{s} A_{s}^{(+)}\left\{-\beta\left[1+(-)^{s} \frac{\xi}{\epsilon}\left(\frac{\beta}{2 \alpha} \frac{\gamma_{-}(-\alpha, \beta)}{\gamma_{+}(\alpha, \beta)} I_{2}+\frac{1}{2} g(-\infty)\right)\right] u_{+}^{s}\right. \\
& \left.\quad+\epsilon\left[1+(-)^{s} \frac{\xi}{\epsilon}\left(\frac{\beta}{2 \alpha} \frac{\gamma_{-}(-\alpha, \beta)}{\gamma_{+}(\alpha, \beta)} I_{2}-\frac{1}{2} g(-\infty)\right)\right] u_{-}^{s}\right\}, \\
& \left(\psi(x)_{\sigma=+}\right)^{r e f l} \\
= & \mathrm{e}^{-i \epsilon \tau-\beta x} \gamma_{+}(\alpha,-\beta) \sum_{s} A_{s}^{(+)}\left\{\beta\left[1+(-)^{s} \frac{\xi}{\epsilon}\left(-\frac{\beta}{2 \alpha} \frac{\gamma_{-}(-\alpha,-\beta)}{\gamma_{+}(\alpha,-\beta)} I_{2}+\frac{1}{2} g(-\infty)\right)\right] u_{+}^{s}\right. \\
& \left.+\epsilon\left[1+(-)^{s} \frac{\xi}{\epsilon}\left(-\frac{\beta}{2 \alpha} \frac{\gamma_{-}(-\alpha,-\beta)}{\gamma_{+}(\alpha,-\beta)} I_{2}-\frac{1}{2} g(-\infty)\right)\right] u_{-}^{s}\right\},
\end{aligned}
$$

\footnotetext{
* This boundary condition is referred to as Type II in ref.[8].
} 
and

$$
\left(\psi(x)_{\sigma=-}\right)^{\text {asym }}=\left.\left(\psi(x)_{\sigma=+}\right)^{\text {asym }}\right|_{\left(\alpha, \beta, \epsilon, I_{1}, I_{2}\right) \rightarrow\left(-\alpha,-\beta,-\epsilon, I_{1}^{*}, I_{2}^{*}\right)},
$$

where

$$
\begin{aligned}
& I_{1}=\int_{-\infty}^{\infty} d x g^{\prime}(x) \phi_{-}^{(-\alpha)}(x) \phi_{+}^{(+\alpha)}(x), \\
& I_{2}=\int_{-\infty}^{\infty} d x g^{\prime}(x) \phi_{-}^{(+\alpha)}(x) \phi_{+}^{(+\alpha)}(x) .
\end{aligned}
$$

From these, the asymptotic forms of the vector and axial-vector currents defined by

$$
j_{V}^{\mu}=\bar{\psi} \gamma^{\mu} \psi, \quad j_{A}^{\mu}=\bar{\psi} \gamma^{\mu} \gamma_{5} \psi
$$

are (see Appendix)

$$
\begin{aligned}
& \left(j_{V, \sigma}^{3}\right)^{\text {trans }}=2 \epsilon \sqrt{\epsilon^{2}-\xi^{2}} \sum_{s}\left|A_{s}^{(\sigma)}\right|^{2}, \\
& \left(j_{A, \sigma}^{3}\right)^{\text {trans }}=2 \epsilon^{2} \sum_{s}(-)^{s+1}\left|A_{s}^{(\sigma)}\right|^{2} \\
& \left(j_{V, \sigma}^{3}\right)_{I I}^{i n c}=2 \epsilon^{2}\left|\gamma_{+}(\alpha, \beta)\right|^{2} \sum_{s}\left|A_{s}^{(\sigma)}\right|^{2}\left[1-\sigma(-)^{s+1} \delta^{i n c}\right], \\
& \left(j_{A, \sigma}^{3}\right)_{I I}^{i n c}=2 \epsilon^{2}\left|\gamma_{+}(\alpha, \beta)\right|^{2} \sum_{s}(-)^{s+1}\left|A_{s}^{(\sigma)}\right|^{2}\left[1-\sigma(-)^{s+1} \delta^{i n c}\right], \\
& \left(j_{V, \sigma}^{3}\right)_{I I}^{r e f l}=-2 \epsilon^{2}\left|\gamma_{+}(\alpha,-\beta)\right|^{2} \sum_{s}\left|A_{s}^{(\sigma)}\right|^{2}\left[1+\sigma(-)^{s+1} \delta^{r e f l}\right], \\
& \left(j_{A, \sigma}^{3}\right)_{I I}^{r e f l}=2 \epsilon^{2}\left|\gamma_{+}(\alpha,-\beta)\right|^{2} \sum_{s}(-)^{s+1}\left|A_{s}^{(\sigma)}\right|^{2}\left[1+\sigma(-)^{s+1} \delta^{r e f l}\right],
\end{aligned}
$$

where the corrections by the $\mathrm{CP}$ violation are

$$
\begin{aligned}
\delta^{i n c} & =\frac{\xi}{2 \sqrt{\epsilon^{2}-\xi^{2}}}\left(\frac{\gamma_{-}(-\alpha, \beta)}{\gamma_{+}(\alpha, \beta)} I_{2}+\text { c.c. }\right), \\
\delta^{r e f l} & =\frac{\xi}{2 \sqrt{\epsilon^{2}-\xi^{2}}}\left(\frac{\gamma_{-}(-\alpha,-\beta)}{\gamma_{+}(\alpha,-\beta)} I_{2}+\text { c.c. }\right) .
\end{aligned}
$$


The chiral currents defined by

$$
j_{L}^{\mu}=\frac{1}{2}\left(j_{V}^{\mu}-j_{A}^{\mu}\right), \quad j_{R}^{\mu}=\frac{1}{2}\left(j_{V}^{\mu}+j_{A}^{\mu}\right)
$$

are

$$
\begin{aligned}
& \left(j_{L, \sigma}^{3}\right)^{\text {trans }}=\epsilon\left(\sqrt{\epsilon^{2}-\xi^{2}}-\epsilon\right)\left|A_{1}^{(\sigma)}\right|^{2}+\epsilon\left(\sqrt{\epsilon^{2}-\xi^{2}}+\epsilon\right)\left|A_{2}^{(\sigma)}\right|^{2}, \\
& \left(j_{R, \sigma}^{3}\right)^{\text {trans }}=\epsilon\left(\sqrt{\epsilon^{2}-\xi^{2}}+\epsilon\right)\left|A_{1}^{(\sigma)}\right|^{2}+\epsilon\left(\sqrt{\epsilon^{2}-\xi^{2}}-\epsilon\right)\left|A_{2}^{(\sigma)}\right|^{2}, \\
& \left(j_{L, \sigma}^{3}\right)^{i n c}=2 \epsilon^{2}\left|\gamma_{+}(\alpha, \beta)\right|^{2}\left|A_{2}^{(\sigma)}\right|^{2}\left(1+\sigma \delta^{i n c}\right), \\
& \left(j_{R, \sigma}^{3}\right)^{i n c}=2 \epsilon^{2}\left|\gamma_{+}(\alpha, \beta)\right|^{2}\left|A_{1}^{(\sigma)}\right|^{2}\left(1-\sigma \delta^{i n c}\right), \\
& \left(j_{L, \sigma}^{3}\right)^{r e f l}=-2 \epsilon^{2}\left|\gamma_{+}(\alpha,-\beta)\right|^{2}\left|A_{1}^{(\sigma)}\right|^{2}\left(1+\sigma \delta^{r e f l}\right), \\
& \left(j_{R, \sigma}^{3}\right)^{r e f l}=-2 \epsilon^{2}\left|\gamma_{+}(\alpha,-\beta)\right|^{2}\left|A_{2}^{(\sigma)}\right|^{2}\left(1-\sigma \delta^{r e f l}\right) .
\end{aligned}
$$

With these currents, one can compute the transmission and reflection coefficients, $T^{(\sigma)}$ and $R^{(\sigma)}$, respectively. From the vector currents, we have

$$
\begin{aligned}
& T^{(\sigma)}=\frac{\left(j_{V, \sigma}^{3}\right)^{\text {trans }}}{\left(j_{V, \sigma}^{3}\right)^{\text {inc }}}=T^{(0)}\left[1+\frac{\sum_{s}(-)^{s+1}\left|A_{s}^{(\sigma)}\right|^{2}}{\sum_{s}\left|A_{s}^{(\sigma)}\right|^{2}} \sigma \delta^{i n c}\right] \\
& R^{(\sigma)}=-\frac{\left(j_{V, \sigma}^{3}\right)^{r e f l}}{\left(j_{V, \sigma}^{3}\right)^{i n c}}=R^{(0)}\left[1+\frac{\sum_{s}(-)^{s+1}\left|A_{s}^{(\sigma)}\right|^{2}}{\sum_{s}\left|A_{s}^{(\sigma)}\right|^{2}} \sigma\left(\delta^{i n c}+\delta^{r e f l}\right)\right]
\end{aligned}
$$

where

$$
\begin{aligned}
& T^{(0)}=\frac{\alpha}{\beta} \frac{1}{\left|\gamma_{+}(\alpha, \beta)\right|^{2}}, \\
& R^{(0)}=\left|\frac{\gamma_{+}(\alpha,-\beta)}{\gamma_{+}(\alpha, \beta)}\right|^{2},
\end{aligned}
$$

are those in the absence of the perturbation. As shown in Appendix, the unitarity 
holds:

$$
T^{(\sigma)}+R^{(\sigma)}=T^{(0)}+R^{(0)}=1 .
$$

The differences in these coefficients between the particle and the anti-particle would vanish, even in the presence of $\mathrm{CP}$ violation, upon averaged over the initial mixed states such as the thermal equilibrium. This is because the difference has a factor, $\frac{\sum_{s}(-)^{s+1}\left|A_{s}^{(\sigma)}\right|^{2}}{\sum_{s}\left|A_{s}^{(\sigma)}\right|^{2}}$, in which $A_{s}^{(\sigma)}$ is representation-dependent and can take any value in a mixed state. Therefore, it is expected that there would be no net (vectorlike) particle current through the bubble wall.

The transmission and reflection coefficients for the chiral fermion are defined as

$$
\begin{aligned}
& T_{L \rightarrow L(R)}^{(\sigma)}=\left.\left(j_{L(R), \sigma}^{3}\right)^{\text {trans }}\right|_{A_{1}^{\sigma}=0} /\left(j_{L, \sigma}^{3}\right)^{i n c}, \\
& T_{R \rightarrow L(R)}^{(\sigma)}=\left.\left(j_{L(R), \sigma}^{3}\right)^{\text {trans }}\right|_{A_{2}^{\sigma}=0} /\left(j_{R, \sigma}^{3}\right)^{i n c}, \\
& R_{L(R) \rightarrow R(L)}^{(\sigma)}=-\left(j_{R(L), \sigma}^{3}\right)^{r e f l} /\left(j_{L(R), \sigma}^{3}\right)^{i n c} .
\end{aligned}
$$

If we denote $R(T)=R^{(+)}\left(T^{(+)}\right)$and $\bar{R}(\bar{T})=R^{(-)}\left(T^{(-)}\right)$, we have

$$
\begin{aligned}
& T_{L \rightarrow L}=\bar{T}_{R \rightarrow R}=\frac{\sqrt{\epsilon^{2}-\xi^{2}}+\epsilon}{2 \epsilon\left|\gamma_{+}(\alpha, \beta)\right|^{2}}\left(1-\delta^{i n c}\right), \\
& T_{L \rightarrow R}=\bar{T}_{R \rightarrow L}=\frac{\sqrt{\epsilon^{2}-\xi^{2}}-\epsilon}{2 \epsilon\left|\gamma_{+}(\alpha, \beta)\right|^{2}}\left(1-\delta^{i n c}\right), \\
& T_{R \rightarrow L}=\bar{T}_{L \rightarrow R}=\frac{\sqrt{\epsilon^{2}-\xi^{2}}-\epsilon}{2 \epsilon\left|\gamma_{+}(\alpha, \beta)\right|^{2}}\left(1+\delta^{i n c}\right), \\
& T_{R \rightarrow R}=\bar{T}_{L \rightarrow L}=\frac{\sqrt{\epsilon^{2}-\xi^{2}}+\epsilon}{2 \epsilon\left|\gamma_{+}(\alpha, \beta)\right|^{2}}\left(1+\delta^{i n c}\right), \\
& R_{L \rightarrow R}=\bar{R}_{R \rightarrow L}=\left|\frac{\gamma_{+}(\alpha,-\beta)}{\gamma_{+}(\alpha, \beta)}\right|^{2}\left(1-\delta^{i n c}-\delta^{r e f l}\right), \\
& R_{R \rightarrow L}=\bar{R}_{L \rightarrow R}=\left|\frac{\gamma_{+}(\alpha,-\beta)}{\gamma_{+}(\alpha, \beta)}\right|^{2}\left(1+\delta^{i n c}+\delta^{r e f l}\right) .
\end{aligned}
$$


Among these, the following unitarity relations hold:

$$
\begin{aligned}
& T_{L \rightarrow L}+T_{L \rightarrow R}+R_{L \rightarrow R}=1, \\
& T_{R \rightarrow L}+T_{R \rightarrow R}+R_{R \rightarrow L}=1 .
\end{aligned}
$$

The difference between a particle and its anti-particle in the coefficients is, for example,

$$
\Delta R \equiv R_{R \rightarrow L}-\bar{R}_{R \rightarrow L}=2 R^{(0)}\left(\delta^{i n c}+\delta^{r e f l}\right)=-2 T^{(0)} \delta^{i n c},
$$

where we used $\delta^{i n c}+R^{(0)} \delta^{r e f l}=0$ (see Appendix). This suggests that we would have nonzero chiral-fermion current through the bubble wall even for the initial state in the equilibrium, if there is a nontrivial CP violation. (By 'nontrivial', we mean that $g(x)$ does not proportional to $f(x)$, since in that case the CP-angle can be removed by a constant bi-unitary transformation of the fermions, or explicitly, $\delta^{i n c}=\delta^{r e f l}=0$, as shown in Appendix.)

\section{Application to the Kink-Type Bubble Wall}

The prescription developed in the previous section is applicable to the situation where the bubble formed in the first-order phase transition is moving with a constant velocity. The profile and motion of such a bubble would be determined by analyzing the detail of dynamics of the phase transition. Far from the wall, deep in the symmetric/broken phase, the Higgs field is expected to have the

vanishing/nonvanishing vacuum expectation value in the uniform vacuum. (This corresponds to the condition (2.9).) The simplest situation may be that in which the profile has the shape of the kink. This case, without CP violation, was studied in ref.[8]. Now we apply our procedure to that case, and evaluate the transmission and reflection coefficients. 
Following [8], we suppose that the bubble-wall profile is given by

$$
f(x)=\frac{1+\tanh x}{2}
$$

Then the solutions to the Klein-Gordon-type equation, (2.17), are expressed in terms of the hypergeometric functions:

$$
\begin{aligned}
& \phi_{ \pm}^{(+\alpha)}(y)=y^{-\alpha / 2}(1-y)^{\beta / 2} F\left(\frac{-\alpha+\beta \mp \xi}{2}+1, \frac{-\alpha+\beta \pm \xi}{2},-\alpha+1 ; y\right), \\
& \phi_{ \pm}^{(-\alpha)}(y)=y^{\alpha / 2}(1-y)^{\beta / 2} F\left(\frac{\alpha+\beta \mp \xi}{2}+1, \frac{\alpha+\beta \pm \xi}{2}, \alpha+1 ; y\right)=\left(\phi_{ \pm}^{(+\alpha)}(y)\right)^{*}
\end{aligned}
$$

where we have changed the variable as $y=(1-\tanh x) / 2{ }^{\star}$ These have the desired asymptotic behaviors as (2.18) and (2.19). Now $\gamma_{ \pm}(\alpha, \beta)$ are explicitly given by

$$
\gamma_{ \pm}(\alpha, \beta)=\frac{\Gamma(-\alpha+1) \Gamma(-\beta)}{\Gamma\left(\frac{-\alpha-\beta \pm \xi}{2}\right) \Gamma\left(\frac{-\alpha-\beta \mp \xi}{2}+1\right)}
$$

The transmission and reflection coefficients without the $\mathrm{CP}$ violation are

$$
\begin{aligned}
T^{(0)} & =\frac{\sin (\pi \alpha) \sin (\pi \beta)}{\sin \left[\frac{\pi}{2}(\alpha+\beta+\xi)\right] \sin \left[\frac{\pi}{2}(\alpha+\beta-\xi)\right]}, \\
R^{(0)} & =\frac{\sin \left[\frac{\pi}{2}(\alpha-\beta+\xi)\right] \sin \left[\frac{\pi}{2}(\alpha-\beta-\xi)\right]}{\sin \left[\frac{\pi}{2}(\alpha+\beta+\xi)\right] \sin \left[\frac{\pi}{2}(\alpha+\beta-\xi)\right]}
\end{aligned}
$$

The effects of the CP violation can be evaluated, once the functional form of $g(x)$ is given. One of the quantities we concern may be the difference in the reflection/transmission coefficients between the particle and anti-particle. As mentioned above, the vectorlike one would vanish after averaging over the initial mixed state. However, the chiral one, which is given by (2.35), would survive even for the initial state in the equilibrium.

\footnotetext{
$\star$ The parameters $\alpha, \beta$ and $\xi$ in ref.[8] are halves of ours.
} 
We have executed numerical calculations for various $g(x)$. Here we present the results for two typical cases in which $g^{\prime}(x)$ has different behaviors around the wall surface, i.e., $g(x)=\Delta \theta \cdot f(x)^{2}$ and $g(x)=\Delta \theta \frac{d}{d x} f(x)$, where $\Delta \theta$ characterizes the magnitude of $\mathrm{CP}$ violation. We carried out the numerical calculation of $\Delta R$ at several energies. The $a$-dependence of $\Delta R / \Delta \theta$ for $g(x)=\Delta \theta \cdot f(x)^{2}$ is summarized in Table 1. The $E^{*}$-dependence of $\Delta R / \Delta \theta$ is plotted in Figs. 1 and 2 for the two choices of $g(x)$ for several $a$. From these, we find the following:

(1) The sign of $\Delta R$ varies not only with a but also with the functional form of $g(x)$.

(2) The absolute value of $\Delta R$ rapidly decreases as the energy of the incident particle increases. This feature may be independent of the choice of $g(x)$, since the $g(x)$-independent factor of $\Delta R$ is very small in the region far from $E^{*} \sim m_{0}$.

(3) The degree of the decrease of $|\Delta R|$ seems to be weakened as a increases, i.e. the bubble wall becomes thinner.

\section{Conclusions}

We have developed a general prescription to solve the Dirac equation in a bubble-wall background with $\mathrm{CP}$ violation, to the first order of $\mathrm{CP}$ violation. The transmission and reflection coefficients are obtained to this order, in terms of the functions of the energy, the mass in the broken phase and the wall thickness, which are extracted from the continuation of the solution to the second-order Klein-Gordon-like equation. It is found, irrespectively of the detailed form of the bubble-wall profile, that for the vectorlike current, the effect of $\mathrm{CP}$ violation will be canceled, when averaged over an initial mixed state such as thermal equilibrium, and that the net chiral current through the bubble wall would remain. Since the relation between the chemical potentials of the left- and right-handed quarks in the symmetric phase is different from that in the broken phase ${ }^{[10]}$, this would leave net baryon number after the phase transition. 
We have applied our prescription to the simple example in which the profile of the bubble wall is of the kink type. This situation may be realized when the phase transition proceeds slowly with degenerate minima of the free energy. Some numerical results are obtained in the case where the CP-violating angle has nontrivial spatial dependence. $\Delta R$, which measures the difference in the reflection probabilities between the chiral fermion and anti-fermion, is evaluated for various energies and wall thickness, assuming some typical forms of $g(x)$. The results suggest that irrespectively of the choice of $g(x),|\Delta R|$ rapidly decreases as the energy of incident fermions increases. Further the degree of decreasing seems to be larger as the wall becomes thicker.

In order to determine whether and how much the net baryon number is left after the electroweak phase transition, we must know the detailed dynamics of the phase transition and particle distribution within both phases divided by the bubble wall. The baryon number left in the broken phase may be calculated by taking the average of the net fermion current through the bubble wall from the symmetric to the broken phase, and vice versa. Since the distribution function of the chiral fermions in the broken phase is different from that in the symmetric phase, as shown from the equilibrium analysis in [10], a quantity such as $\Delta R$ will play an important role in estimating the baryon asymmetry of the universe. As far as the cases we have studied are concerned, $\Delta R$ shows no drastic enhancement. On the other hand, even the sign of $\Delta R$ varies with the inverse wall-thickness $a$, as well as with the functional form of $g(x)$. Thus whether the baryon or anti-baryon is left after the electroweak phase transition would be a subtle problem. We would like to remark that $|\Delta \theta|$ does not need to be extremely small in so far as $|g(+\infty)|$ is small enough to satisfy the experimental bound. In fact, in our second example $\left(g(x)=\Delta \theta f^{\prime}(x)\right), g(+\infty)$ vanishes irrespective of the value of $\Delta \theta$. This fact might allow us to build a model which produces sufficient baryon number, by taking a suitable form of $g(x)$.

Our results will be applied to the scenario of the electroweak baryogenesis, which assumes the first-order phase transition. Although much should be revealed 
about the mechanism of $\mathrm{CP}$ violation and the dynamics of the phase transition, such as the order of it and the motion of the bubble wall when it is of first order, the baryon asymmetry of the universe might be generated in the electroweak phase transition era if there is sufficient baryon number current through the bubble wall.

\section{APPENDIX}

In this appendix, we shall derive some equations used in the text.

\section{Unperturbed wave function}

The second-order unperturbed equation (2.17) is written as

$$
D_{\mp} D_{ \pm} \phi_{ \pm}(x)=\epsilon^{2} \phi_{ \pm}(x)
$$

where $\phi(x)$ is either $\phi^{(+\alpha)}(x)$ or $\phi^{(-\alpha)}(x)$ and the operator $D_{ \pm}$is defined by

$$
D_{ \pm} \equiv \mp \frac{d}{d x}+\xi f(x)
$$

Multiplying (A.1) by $D_{ \pm}$, we have

$$
\left(D_{ \pm} D_{\mp}\right)\left(D_{ \pm} \phi_{ \pm}^{(+\alpha)}(x)\right)=\epsilon^{2}\left(D_{ \pm} \phi_{ \pm}^{(+\alpha)}(x)\right) .
$$

Since the independent solutions to this equation are $\phi_{\mp}^{(+\alpha)}(x)$ and $\phi_{\mp}^{(-\alpha)}(x)$, $D_{ \pm} \phi_{ \pm}^{(+\alpha)}(x)$ should be expressed as

$$
D_{ \pm} \phi_{ \pm}^{(+\alpha)}(x)=c_{ \pm}^{(+\alpha)} \phi_{\mp}^{(+\alpha)}(x)+c_{ \pm}^{(-\alpha)} \phi_{\mp}^{(-\alpha)}(x) .
$$

From the behavior of the left-hand side at $x \sim+\infty$;

$$
D_{ \pm} \phi_{ \pm}^{(+\alpha)}(x) \sim(\xi \mp \alpha) \mathrm{e}^{\alpha x}
$$


it follows that $c_{ \pm}^{(+\alpha)}=\xi \mp \alpha$ and $c_{ \pm}^{(-\alpha)}=0$. That is,

$$
\begin{aligned}
& {\left[\mp \frac{d}{d x}+\xi f(x)\right] \phi_{ \pm}^{(-\alpha)}(x)=(\xi \pm \alpha) \phi_{\mp}^{(-\alpha)}(x),} \\
& {\left[\mp \frac{d}{d x}+\xi f(x)\right] \phi_{ \pm}^{(+\alpha)}(x)=(\xi \mp \alpha) \phi_{\mp}^{(+\alpha)}(x) .}
\end{aligned}
$$

In the Dirac representation, the $\gamma$-matrices are represented as

$$
\gamma^{3}=\left(\begin{array}{cc}
0 & \sigma_{3} \\
-\sigma_{3} & 0
\end{array}\right), \quad \gamma^{0}=\left(\begin{array}{cc}
1 & 0 \\
0 & -1
\end{array}\right)
$$

and the eigenspinors of $\gamma^{3}$ satisfying

$$
\gamma^{3} u_{ \pm}^{s}= \pm i u_{ \pm}^{s}, \quad(s=1,2)
$$

are expressed as

$$
u_{ \pm}^{1}=\frac{1}{\sqrt{2}}\left(\begin{array}{c}
1 \\
0 \\
\pm i \\
0
\end{array}\right), \quad u_{ \pm}^{2}=\frac{1}{\sqrt{2}}\left(\begin{array}{c}
0 \\
1 \\
0 \\
\mp i
\end{array}\right)
$$

Then $\gamma^{0} u_{ \pm}^{s}=u_{\mp}^{s}$. The general solution to the unperturbed Dirac equation (2.20) is obtained by multiplying the most general $\psi^{(0)}(x)$ by the operator $\sigma \epsilon \gamma^{0}+i \gamma^{3} \frac{d}{d x}+$ $\xi f(x)$ :

$$
\begin{aligned}
\psi_{0}(x) \equiv\left[\sigma \epsilon \gamma^{0}+i \gamma^{3} \frac{d}{d x}+\xi f(x)\right] \psi^{(0)}(x) \\
=\sum_{s}\left\{\left(A_{+}^{s}\right)^{(-\alpha)}\left[\sigma \epsilon \phi_{+}^{(-\alpha)}(x) u_{-}^{s}+(\xi+\alpha) \phi_{-}^{(-\alpha)}(x) u_{+}^{s}\right]\right. \\
+\left(A_{-}^{s}\right)^{(-\alpha)}\left[\sigma \epsilon \phi_{-}^{(-\alpha)}(x) u_{+}^{s}+(\xi-\alpha) \phi_{+}^{(-\alpha)}(x) u_{-}^{s}\right] \\
+\left(A_{+}^{s}\right)^{(+\alpha)}\left[\sigma \epsilon \phi_{+}^{(+\alpha)}(x) u_{-}^{s}+(\xi-\alpha) \phi_{-}^{(+\alpha)}(x) u_{+}^{s}\right] \\
\left.+\left(A_{-}^{s}\right)^{(+\alpha)}\left[\sigma \epsilon \phi_{-}^{(+\alpha)}(x) u_{+}^{s}+(\xi+\alpha) \phi_{+}^{(+\alpha)}(x) u_{-}^{s}\right]\right\} .
\end{aligned}
$$

Here $\sigma \epsilon \phi_{+}^{(-\alpha)}(x) u_{-}^{s}+(\xi+\alpha) \phi_{-}^{(-\alpha)}(x) u_{+}^{s}$ and $\sigma \epsilon \phi_{-}^{(-\alpha)}(x) u_{+}^{s}+(\xi-\alpha) \phi_{+}^{(-\alpha)}(x) u_{-}^{s}$ are not independent with each other, since the determinant of the coefficients of their 
components vanishes: $\left|\begin{array}{cc}\sigma \epsilon & \xi+\alpha \\ \xi-\alpha & \sigma \epsilon\end{array}\right|=0$. Similarly the functions in the last two lines of (A.6) are not independent. Hence the most general solution is given by putting $\left(A_{-}^{s}\right)^{(-\alpha)}=\left(A_{-}^{s}\right)^{(+\alpha)}=0$; the most general $\psi^{(0)}(x)$ is

$$
\psi^{(0)}(x)=\sum_{s}\left[A_{s}^{(-\alpha)} \phi_{+}^{(-\alpha)}(x)+A_{s}^{(+\alpha)} \phi_{+}^{(+\alpha)}(x)\right] u_{+}^{s}
$$

The wave function satisfying the boundary condition which we adopted in the text is obtained by putting $A_{s}^{(-\sigma \alpha)}=0$.

Green's function

Here we construct the Green's function satisfying

$$
\left[\epsilon^{2}+\frac{d^{2}}{d x^{2}}-\xi^{2} f(x)^{2}+i \xi f^{\prime}(x) \gamma^{3}\right]_{\alpha \gamma} G_{\gamma \beta}\left(x, x^{\prime}\right)=\Delta_{\alpha \gamma} G_{\gamma \beta}\left(x, x^{\prime}\right)=-\delta_{\alpha \beta} \delta\left(x-x^{\prime}\right)
$$

with the boundary condition discussed in the text. Put

$$
\Delta_{ \pm}=\frac{d^{2}}{d x^{2}} \mp \xi f^{\prime}(x)-\xi^{2} f(x)^{2}+\epsilon^{2}
$$

and introduce a unitary matrix defined by

$$
U=\left(u_{+}^{1} u_{-}^{1} u_{+}^{2} u_{-}^{2}\right)
$$

Then, since $\Delta$ is diagonalized as

$$
\Delta=U\left(\begin{array}{cccc}
\Delta_{+} & & & \\
& \Delta_{-} & & \\
& & \Delta_{+} & \\
& & & \Delta_{-}
\end{array}\right) U^{-1}
$$

the Green's function is given by

$$
G\left(x, x^{\prime}\right)=U\left(\begin{array}{llll}
G_{+}\left(x, x^{\prime}\right) & & & \\
& G_{-}\left(x, x^{\prime}\right) & & \\
& & G_{+}\left(x, x^{\prime}\right) & \\
& & G_{-}\left(x, x^{\prime}\right)
\end{array}\right) U^{-1}
$$


where $G_{ \pm}\left(x, x^{\prime}\right)$ satisfies

$$
\Delta_{ \pm} G_{ \pm}\left(x, x^{\prime}\right)=-\delta\left(x-x^{\prime}\right)
$$

with the same boundary condition as $G\left(x, x^{\prime}\right)$. One can construct the Green's function $G_{ \pm}\left(x, x^{\prime}\right)$ following the standard method ${ }^{[9]}$. If $u_{ \pm}(x)\left(v_{ \pm}(x)\right)$ are solutions to $\Delta_{ \pm} u_{ \pm}(x)=0\left(\Delta_{ \pm} v_{ \pm}(x)=0\right)$ and satisfy the boundary condition imposed on $G\left(x, x^{\prime}\right)$ at $x \rightarrow-\infty(x \rightarrow+\infty)$, then the Green's function is expressed as

$$
G_{ \pm}\left(x, x^{\prime}\right)= \begin{cases}-\frac{1}{W\left(u_{ \pm}, v_{ \pm}\right)} u_{ \pm}(x) v_{ \pm}\left(x^{\prime}\right), & \left(x<x^{\prime}\right) \\ -\frac{1}{W\left(u_{ \pm}, v_{ \pm}\right)} u_{ \pm}\left(x^{\prime}\right) v_{ \pm}(x), & \left(x^{\prime}<x\right)\end{cases}
$$

where $W(u, v)$ is the Wronskian defined by

$$
W\left(u_{ \pm}, v_{ \pm}\right)=u_{ \pm}(x) \frac{d}{d x} v_{ \pm}(x)-v_{ \pm}(x) \frac{d}{d x} u_{ \pm}(x)=\text { const }
$$

For the boundary condition in question, there exists only right-moving wave at $x \sim+\infty$ so that we should choose, for each $\sigma$,

$$
v_{ \pm}(x)=\phi_{ \pm}^{(+\sigma \alpha)}(x)
$$

while we take the most general choice for $u_{ \pm}(x)$;

$$
u_{ \pm}(x)=\phi_{ \pm}^{(-\sigma \alpha)}(x)+c_{ \pm}^{(\sigma)} \phi_{ \pm}^{(+\sigma \alpha)}(x) .
$$

where $c_{ \pm}^{(\sigma)}$ is some constant. (The overall normalization is irrelevant, since the product of $u$ and $v$ is divided by the Wronskian in (A.11).) By use of the asymptotic forms of $\phi_{ \pm}(x)$ at $x \sim+\infty$,

$$
W\left(u_{ \pm}, v_{ \pm}\right)=2 \sigma \alpha
$$

Thus the Green's function $G_{ \pm}\left(x, x^{\prime}\right)$ is

$$
G_{ \pm}^{(\sigma)}\left(x, x^{\prime}\right)= \begin{cases}-\frac{\sigma}{2 \alpha}\left[\phi_{ \pm}^{(-\sigma \alpha)}(x)+c_{ \pm}^{(\sigma)} \phi_{ \pm}^{(+\sigma \alpha)}(x)\right] \phi_{ \pm}^{(+\sigma \alpha)}\left(x^{\prime}\right), & \left(x<x^{\prime}\right) \\ -\frac{\sigma}{2 \alpha}\left[\phi_{ \pm}^{(-\sigma \alpha)}\left(x^{\prime}\right)+c_{ \pm}^{(\sigma)} \phi_{ \pm}^{(+\sigma \alpha)}\left(x^{\prime}\right)\right] \phi_{ \pm}^{(+\sigma \alpha)}(x), & \left(x^{\prime}<x\right)\end{cases}
$$

$D W B A$ to the first order 
As explained in Section 2.2, the function $\psi_{\epsilon}(x)$ in the Ansatz (2.7) is given by

$$
\psi_{\epsilon}(x)=\psi^{(0)}(x)+\psi^{(1)}(x)
$$

where $\psi^{(0)}(x)$ is a solution to the unperturbed equation with the boundary condition above, that is,

$$
\psi^{(0)}(x)=\sum_{s} A_{s}^{(\sigma)} \phi_{+}^{(+\sigma \alpha)}(x) u_{+}^{s},
$$

and $\psi^{(1)}(x)$ is

$$
\psi^{(1)}(x)=\int d x^{\prime} G\left(x, x^{\prime}\right) V\left(x^{\prime}\right) \psi^{(0)}\left(x^{\prime}\right)
$$

Noting that in the Dirac representation, $\gamma_{5}=\left(\begin{array}{ll}0 & 1 \\ 1 & 0\end{array}\right)$ so that

$$
\gamma_{5} \gamma^{3} u_{ \pm}^{s}=(-)^{s} u_{\mp}^{s}
$$

and $V(x)$ is

$$
V(x)=-\xi g^{\prime}(x) \gamma_{5} \gamma^{3}
$$

the integrand of (A.16) is written as

$$
G\left(x, x^{\prime}\right) V\left(x^{\prime}\right) \psi^{(0)}\left(x^{\prime}\right)=-\xi G_{-}^{(\sigma)}\left(x, x^{\prime}\right) g^{\prime}\left(x^{\prime}\right) \sum_{s}(-)^{s} A_{s}^{(\sigma)} \phi_{+}^{(+\sigma \alpha)}\left(x^{\prime}\right) u_{-}^{s} .
$$

Therefore $\psi^{(1)}(x)$ is

$$
\begin{aligned}
\psi^{(1)}(x)= & \frac{\sigma \xi}{2 \alpha} \sum_{s}(-)^{s} A_{s}^{(\sigma)} u_{-}^{s} \\
& \times\left\{\left[\phi_{-}^{(-\sigma \alpha)}(x)+c_{-}^{(\sigma)} \phi_{-}^{(+\sigma \alpha)}(x)\right] \int_{x}^{\infty} d x^{\prime} g^{\prime}\left(x^{\prime}\right) \phi_{-}^{(+\sigma \alpha)}\left(x^{\prime}\right) \phi_{+}^{(+\sigma \alpha)}\left(x^{\prime}\right)\right. \\
& \left.+\phi_{-}^{(+\sigma \alpha)}(x) \int_{-\infty}^{x} d x^{\prime} g^{\prime}\left(x^{\prime}\right)\left[\phi_{-}^{(-\sigma \alpha)}\left(x^{\prime}\right)+c_{-}^{(\sigma)} \phi_{-}^{(+\sigma \alpha)}\left(x^{\prime}\right)\right] \phi_{+}^{(+\sigma \alpha)}\left(x^{\prime}\right)\right\}
\end{aligned}
$$


and its asymptotic forms are

$$
\begin{aligned}
\left(\psi^{(1)}(x)\right)^{+\infty}= & \frac{\xi}{2 \alpha} \mathrm{e}^{\alpha x}\left(I_{1}+c_{-}^{(+)} I_{2}\right) \sum_{s}(-)^{s} A_{s}^{(+)} u_{-}^{s}, \\
\left(\psi^{(1)}(x)\right)^{-\infty}= & \frac{\xi}{2 \alpha} I_{2} \sum_{s}(-)^{s} A_{s}^{(+)} u_{-}^{s} \\
& \times\left[\left(\gamma_{-}(-\alpha, \beta)+c_{-}^{(+)} \gamma_{-}(\alpha, \beta)\right) \mathrm{e}^{\beta x}+\left(\gamma_{-}(-\alpha,-\beta)+c_{-}^{(+)} \gamma_{-}(\alpha,-\beta)\right) \mathrm{e}^{-\beta x}\right],
\end{aligned}
$$

for the positive-energy wave, and

$$
\begin{aligned}
\left(\psi^{(1)}(x)\right)^{+\infty}= & -\frac{\xi}{2 \alpha} \mathrm{e}^{-\alpha x}\left(I_{1}^{*}+c_{-}^{(-)} I_{2}^{*}\right) \sum_{s}(-)^{s} A_{s}^{(-)} u_{-}^{s}, \\
\left(\psi^{(1)}(x)\right)^{-\infty}= & -\frac{\xi}{2 \alpha} I_{2}^{*} \sum_{s}(-)^{s} A_{s}^{(-)} u_{-}^{s} \\
& \times\left[\left(\gamma_{-}(\alpha, \beta)+c_{-}^{(-)} \gamma_{-}(-\alpha, \beta)\right) \mathrm{e}^{\beta x}+\left(\gamma_{-}(\alpha,-\beta)+c_{-}^{(-)} \gamma_{-}(-\alpha,-\beta)\right) \mathrm{e}^{-\beta x}\right],
\end{aligned}
$$

for the negative-energy wave, where

$$
\begin{aligned}
& I_{1}=\int_{-\infty}^{\infty} d x g^{\prime}(x) \phi_{-}^{(-\alpha)}(x) \phi_{+}^{(+\alpha)}(x), \\
& I_{2}=\int_{-\infty}^{\infty} d x g^{\prime}(x) \phi_{-}^{(+\alpha)}(x) \phi_{+}^{(+\alpha)}(x) .
\end{aligned}
$$

With these asymptotic forms and those of $\psi^{(0)}(x)$,

$$
\begin{aligned}
& \left(\psi^{(0)}(x)\right)^{+\infty}=\mathrm{e}^{\sigma \alpha x} \sum_{s} A_{s}^{(\sigma)} u_{+}^{s}, \\
& \left(\psi^{(0)}(x)\right)^{-\infty}=\sum_{s} A_{s}^{(\sigma)} u_{+}^{s}\left[\gamma_{+}(\sigma \alpha, \beta) \mathrm{e}^{\beta x}+\gamma_{+}(\sigma \alpha,-\beta) \mathrm{e}^{-\beta x}\right],
\end{aligned}
$$

we can compute the asymptotic forms of the perturbed wave function using (2.15);

$$
\psi(x)=\mathrm{e}^{-i \sigma \epsilon \tau}\left\{\left[\sigma \epsilon \gamma^{0}+i \gamma^{3} \frac{d}{d x}+\xi f(x)\right]\left[\psi^{(0)}(x)+\psi^{(1)}(x)\right]-i \xi g(x) \gamma_{5} \psi^{(0)}(x)\right\}
$$


For the positive-energy wave, we have

$$
\begin{aligned}
\left(\psi(x)_{\sigma=+}\right)^{\text {trans }}= & \mathrm{e}^{-i \epsilon \tau+\alpha x} \sum_{s} A_{s}^{(+)}\left[1+(-)^{s} \frac{\xi}{\epsilon} \frac{\xi+\alpha}{2 \alpha} c_{-}^{(+)} I_{2}\right] \\
\times & \left\{(\xi-\alpha)\left[1+(-)^{s} \frac{\xi}{\epsilon} \frac{\xi+\alpha}{2 \alpha} I_{1}\right] u_{+}^{s}\right. \\
& \left.+\epsilon\left[1+(-)^{s} \frac{\xi}{\epsilon}\left(\frac{\xi+\alpha}{2 \alpha} I_{1}-g(+\infty)\right)\right] u_{-}^{s}\right\}, \\
\left(\psi(x)_{\sigma=+}\right)^{i n c}= & \mathrm{e}^{-i \epsilon \tau+\beta x} \gamma_{+}(\alpha, \beta) \sum_{s} A_{s}^{(+)}\left[1+(-)^{s} \frac{\xi}{\epsilon} \frac{\beta}{2 \alpha} \frac{\gamma_{-}(\alpha, \beta)}{\gamma_{+}(\alpha, \beta)} c_{-}^{(+)} I_{2}\right] \\
\times & \left\{-\beta\left[1+(-)^{s} \frac{\xi}{\epsilon} \frac{\beta}{2 \alpha} \frac{\gamma_{-}(-\alpha, \beta)}{\gamma_{+}(\alpha, \beta)} I_{2}\right] u_{+}^{s}\right. \\
& \left.+\epsilon\left[1+(-)^{s} \frac{\xi}{\epsilon}\left(\frac{\beta}{2 \alpha} \frac{\gamma_{-}(-\alpha, \beta)}{\gamma_{+}(\alpha, \beta)} I_{2}-g(-\infty)\right)\right] u_{-}^{s}\right\}, \\
\left(\psi(x)_{\sigma=+}\right)^{r e f l}= & \mathrm{e}^{-i \epsilon \tau-\beta x} \gamma_{+}(\alpha,-\beta) \sum_{s} A_{s}^{(+)}\left[1-(-)^{s} \frac{\xi}{\epsilon} \frac{\beta}{2 \alpha} \frac{\gamma_{-}(\alpha,-\beta)}{\gamma_{+}(\alpha,-\beta)} c_{-}^{(+)} I_{2}\right] \\
\times & \left\{\beta\left[1-(-)^{s} \frac{\xi}{\epsilon} \frac{\beta}{2 \alpha} \frac{\gamma_{-}(-\alpha,-\beta)}{\gamma_{+}(\alpha,-\beta)} I_{2}\right] u_{+}^{s}\right. \\
& \left.+\epsilon\left[1+(-)^{s} \frac{\xi}{\epsilon}\left(-\frac{\beta}{2 \alpha} \frac{\gamma_{-}(-\alpha,-\beta)}{\gamma_{+}(\alpha,-\beta)} I_{2}-g(-\infty)\right)\right] u_{-}^{s}\right\} .
\end{aligned}
$$

The negative-energy wave is related to the positive one by

$$
\left(\psi(x)_{\sigma=-}\right)^{\text {asym }}=\left.\left(\psi(x)_{\sigma=+}\right)^{\text {asym }}\right|_{\left(\alpha, \beta, \epsilon, I_{1}, I_{2}\right) \rightarrow\left(-\alpha,-\beta,-\epsilon, I_{1}^{*}, I_{2}^{*}\right)} .
$$

When one of the relations (A.3),

$$
\left[\frac{d}{d x}+\xi f(x)\right] \phi_{-}^{(+\alpha)}(x)=(\xi+\alpha) \phi_{+}^{(+\alpha)}(x),
$$

is evaluated at $x \sim-\infty$, it produces

$$
\beta \gamma_{-}(\alpha, \beta) \mathrm{e}^{\beta x}-\beta \gamma_{-}(\alpha,-\beta) \mathrm{e}^{-\beta x}=(\xi+\alpha)\left[\gamma_{+}(\alpha, \beta) \mathrm{e}^{\beta x}+\gamma_{+}(\alpha,-\beta) \mathrm{e}^{-\beta x}\right],
$$

or

$$
\begin{aligned}
& \beta \gamma_{-}(\alpha, \beta)=(\xi+\alpha) \gamma_{+}(\alpha, \beta), \\
& \beta \gamma_{-}(\alpha,-\beta)=-(\xi+\alpha) \gamma_{+}(\alpha,-\beta) .
\end{aligned}
$$


These relations lead to

$$
\frac{\xi+\alpha}{2 \alpha}=\frac{\beta}{2 \alpha} \frac{\gamma_{-}(\alpha, \beta)}{\gamma_{+}(\alpha, \beta)}=-\frac{\beta}{2 \alpha} \frac{\gamma_{-}(\alpha,-\beta)}{\gamma_{+}(\alpha,-\beta)}
$$

Hence all the factors including $c_{-}^{(+)} I_{2}$ in (A.19) are the same, so that they are absorbed in the arbitrary coefficients $A_{s}^{( \pm)}$by redefining them as

$$
\begin{aligned}
& A_{s}^{(+)}\left[1+(-)^{s} \frac{\xi}{\epsilon} \frac{\xi+\alpha}{2 \alpha} c_{-}^{(+)} I_{2}\right]\left[1-(-)^{s} \frac{\xi}{\epsilon} \frac{1}{2} g(-\infty)\right] \rightarrow A_{s}^{(+)}, \\
& A_{s}^{(-)}\left[1+(-)^{s} \frac{\xi}{\epsilon} \frac{\xi-\alpha}{2 \alpha} c_{-}^{(-)} I_{2}^{*}\right]\left[1+(-)^{s} \frac{\xi}{\epsilon} \frac{1}{2} g(-\infty)\right] \rightarrow A_{s}^{(-)},
\end{aligned}
$$

where the last factor of the left-hand side is put for later convenience. This gives the result $(2.23)$.

\section{Currents and unitarity}

Now it is straightforward to calculate the asymptotic forms of the various currents, with the wave function (2.23). The straightforward calculation yields the same result as (2.26) except for $\left(j_{V, \sigma}^{3}\right)^{\text {trans }}$ and $\left(j_{A, \sigma}^{3}\right)^{\text {trans }}$, which are

$$
\begin{aligned}
& \left(j_{V, \sigma}^{3}\right)^{\text {trans }}=-2 i \epsilon \alpha \sum_{s}\left|A_{s}^{(\sigma)}\right|^{2}\left\{1+(-)^{s} \sigma \frac{\xi}{\epsilon}\left[-g(+\infty)+g(-\infty)+\left(\frac{\xi+\alpha}{2 \alpha} I_{1}+\text { c.c. }\right)\right]\right\}, \\
& \left(j_{A, \sigma}^{3}\right)^{\text {trans }}=2 \epsilon^{2} \sum_{s}(-)^{s}\left|A_{s}^{(\sigma)}\right|^{2}\left\{1+(-)^{s} \sigma \frac{\xi}{\epsilon}\left[-g(+\infty)+g(-\infty)+\left(\frac{\xi+\alpha}{2 \alpha} I_{1}+\text { c.c. }\right)\right]\right\} .
\end{aligned}
$$

Here one can show that

$$
\frac{\xi+\alpha}{2 \alpha} I_{1}+\text { c.c. }=g(+\infty)-g(-\infty)
$$

so that the result in $(2.26)$ is obtained. Let us prove this equation. Define $\tilde{g}(x)=$ 
$g(x)-g(-\infty)$, then $g^{\prime}(x)=\tilde{g}^{\prime}(x)$ and $\tilde{g}(-\infty)=0$. Integrating by parts, $I_{1}$ becomes

$$
\begin{aligned}
I_{1} & =\int_{-\infty}^{\infty} d x \tilde{g}^{\prime}(x) \phi_{-}^{(-\alpha)}(x) \phi_{+}^{(+\alpha)}(x) \\
= & \left.\tilde{g}(x) \phi_{-}^{(-\alpha)}(x) \phi_{+}^{(+\alpha)}(x)\right|_{-\infty} ^{\infty} \\
& \quad-\int_{-\infty}^{\infty} d x \tilde{g}(x)\left[\phi_{-}^{(-\alpha)^{\prime}}(x) \phi_{+}^{(+\alpha)}(x)+\phi_{-}^{(-\alpha)}(x) \phi_{+}^{(+\alpha)^{\prime}}(x)\right] .
\end{aligned}
$$

From (A.3), the first derivatives of $\phi_{ \pm}(x)$ can be written as

$$
\begin{aligned}
& \phi_{-}^{(-\alpha)^{\prime}}(x)=-\xi f(x) \phi_{-}^{(-\alpha)}(x)+(\xi-\alpha) \phi_{+}^{(-\alpha)}(x), \\
& \phi_{+}^{(+\alpha)^{\prime}}(x)=\xi f(x) \phi_{+}^{(+\alpha)}(x)-(\xi-\alpha) \phi_{-}^{(+\alpha)}(x) .
\end{aligned}
$$

Hence,

$$
I_{1}=\tilde{g}(+\infty)-(\xi-\alpha) \int_{-\infty}^{\infty} d x \tilde{g}(x)\left[\left|\phi_{+}^{(+\alpha)}(x)\right|^{2}-\left|\phi_{-}^{(+\alpha)}(x)\right|^{2}\right]
$$

which leads to

$$
\begin{aligned}
& \frac{\xi+\alpha}{2 \alpha} I_{1}+\text { c.c. } \\
= & \left(\frac{\xi+\alpha}{2 \alpha}+\text { c.c. }\right) \tilde{g}(+\infty)-\left(\frac{\xi^{2}-\alpha^{2}}{2 \alpha}+\text { c.c. }\right) \int_{-\infty}^{\infty} d x \tilde{g}(x)\left[\left|\phi_{+}^{(+\alpha)}(x)\right|^{2}-\left|\phi_{-}^{(+\alpha)}(x)\right|^{2}\right] \\
= & \tilde{g}(+\infty) .
\end{aligned}
$$

Next we shall examine the unitarity. Without the perturbation, the unitarity relation,

$$
T^{(0)}+R^{(0)}=\frac{\alpha}{\beta} \frac{1}{\left|\gamma_{+}(\alpha, \beta)\right|^{2}}+\left|\frac{\gamma_{+}(\alpha,-\beta)}{\gamma_{+}(\alpha, \beta)}\right|^{2}=1,
$$

immediately follows from the current conservation which is derived from the KleinGordon-type equation, (2.17), or in other words, from the fact that the Wronskian 
$W\left(\phi_{+}^{(-\alpha)}, \phi_{+}^{(+\alpha)}\right)$ is independent of $x$. Evaluated at $x \sim+\infty$ and at $x \sim-\infty$, the Wronskian is

$$
W\left(\phi_{+}^{(-\alpha)}, \phi_{+}^{(+\alpha)}\right)=2 \alpha=2 \beta\left(\left|\gamma_{+}(\alpha, \beta)\right|^{2}-\left|\gamma_{+}(\alpha,-\beta)\right|^{2}\right),
$$

which is nothing but (A.22). In the presence of the CP-violation, the sum of the transmission and reflection coefficients is, from (2.29),

$$
T^{(\sigma)}+R^{(\sigma)}=1+\frac{\sum_{s}(-)^{s+1}\left|A_{s}^{(\sigma)}\right|^{2}}{\sum_{s}\left|A_{s}^{(\sigma)}\right|^{2}} \sigma\left(\delta^{i n c}+R^{(0)} \delta^{r e f l}\right) .
$$

Now we can show that

$$
\delta^{i n c}+R^{(0)} \delta^{r e f l}=0
$$

so that the unitarity of the perturbed wave holds. This relations is verified as follows. From the definitions,

$$
\delta^{i n c}+R^{(0)} \delta^{r e f l}=\frac{\xi}{\epsilon} \frac{\beta}{2 \alpha}\left[\left(\frac{\gamma_{-}(-\alpha, \beta)}{\gamma_{+}(\alpha, \beta)}+R^{(0)} \frac{\gamma_{-}(-\alpha,-\beta)}{\gamma_{+}(\alpha,-\beta)}\right)+c . c .\right] .
$$

Here the term in the parentheses is written as

$$
\frac{\gamma_{-}(-\alpha, \beta)}{\gamma_{+}(\alpha, \beta)}+R^{(0)} \frac{\gamma_{-}(-\alpha,-\beta)}{\gamma_{+}(\alpha,-\beta)}=\frac{\gamma_{+}(-\alpha,-\beta) \gamma_{-}(-\alpha, \beta)+\gamma_{+}(-\alpha, \beta) \gamma_{-}(-\alpha,-\beta)}{\left|\gamma_{+}(\alpha, \beta)\right|^{2}}=0
$$

where we have used (A.20) to rewrite $\gamma_{-}$in terms of $\gamma_{+}$in the denominator.

The transmission and reflection coefficients of the chiral fermions are expressed as

$$
\begin{aligned}
& T_{L \rightarrow L}+T_{L \rightarrow R}=T^{(0)}\left(1-\delta^{i n c}\right), \\
& T_{R \rightarrow L}+T_{R \rightarrow R}=T^{(0)}\left(1+\delta^{i n c}\right), \\
& R_{L \rightarrow R}=R^{(0)}\left(1-\delta^{i n c}-\delta^{r e f l}\right), \\
& R_{R \rightarrow L}=R^{(0)}\left(1+\delta^{i n c}+\delta^{r e f l}\right),
\end{aligned}
$$


from which it follows that

$$
\begin{aligned}
& T_{L \rightarrow L}+T_{L \rightarrow R}+R_{L \rightarrow R}=1-\left(\delta^{i n c}+R^{(0)} \delta^{r e f l}\right)=1, \\
& T_{R \rightarrow L}+T_{R \rightarrow R}+R_{R \rightarrow L}=1+\left(\delta^{i n c}+R^{(0)} \delta^{r e f l}\right)=1,
\end{aligned}
$$

because of (A.23).

When $g(x)$ is a linear function of $f(x)$, it is shown that $\delta^{i n c}=\delta^{r e f l}=0$. This is verified as follows. By use of the differential equation (A.1), the integral in which $g^{\prime}(x)$ is replaced with $f^{\prime}(x)$ becomes

$$
\begin{aligned}
\tilde{I}_{2} & \equiv \int_{-\infty}^{\infty} d x f^{\prime}(x) \phi_{-}^{(+\alpha)}(x) \phi_{+}^{(+\alpha)}(x) \\
& =\frac{1}{2 \xi} \int_{-\infty}^{\infty} d x\left[\phi_{-}^{(+\alpha)}(x) \frac{d^{2}}{d x^{2}} \phi_{+}^{(+\alpha)}(x)-\phi_{+}^{(+\alpha)}(x) \frac{d^{2}}{d x^{2}} \phi_{-}^{(+\alpha)}(x)\right] \\
& =\frac{1}{2 \xi}\left[\phi_{-}^{(+\alpha)}(x) \frac{d}{d x} \phi_{+}^{(+\alpha)}(x)-\phi_{+}^{(+\alpha)}(x) \frac{d}{d x} \phi_{-}^{(+\alpha)}(x)\right]_{-\infty}^{\infty} \\
& =-\frac{\beta}{\xi}\left[\gamma_{+}(\alpha, \beta) \gamma_{-}(\alpha,-\beta)-\gamma_{+}(\alpha,-\beta) \gamma_{-}(\alpha, \beta)\right] .
\end{aligned}
$$

Then, with the help of (A.20),

$$
\frac{\gamma_{-}(-\alpha, \beta)}{\gamma_{+}(\alpha, \beta)} \tilde{I}_{2}=-\frac{2 \beta}{\xi}\left|\gamma_{-}(\alpha,-\beta)\right|^{2},
$$

which is purely imaginary, so that $\delta^{i n c}=0$ from the definition in (2.27), and $\delta^{r e f l}=0$ because of (A.23). 


\section{REFERENCES}

1. A. Sakharov, JETP Lett. 5 (1967) 24.

2. G. 't Hooft, Phys. Rev. Lett. 37 (1976) 8;

V. Kuzmin, V. Rubakov and M. Shaposhnikov, Phys. Lett. B155 (1985) 36.

3. D. E. Brahm, Proceedings of the XXVI International Conference on High Energy Physics (1993) 1583, and references therein.

4. M. Kobayashi and T. Maskawa, Prog. Theor. Phys. 49 (1973) 652.

5. N. Turok and J. Zadrozny, Nucl. Phys. B369 (1992) 729.

6. K. Funakubo, A. Kakuto and K. Takenaga, preprint KYUSHU-HET-10-SAGA-HE-50 (1993), to be published in Prog. Theor. Phys. 91 No. 2 (1994).

7. A. Cohen, D. Kaplan and A. Nelson, Nucl. Phys. B373 (1992) 453.

8. A. Ayala, J. Jalilian-Marian, L. McLerran and A. P. Vischer, preprint TPI-MINN-54-MUC-MINN-93/30-T-UMN-TH-1226/93 (1993).

9. See for example, R. Courant and D. Hilbert, Methods of Mathematical Physics ( Interscience, New York ) (1953).

10. M. Fukugita and T. Yanagida, Phys. Lett. B174 (1986) 45; Phys. Rev. D42 (1990) 1285;

J. A. Harvey and M. S. Turner, Phys. Rev. D42 (1990) 3344. 


\section{Table Caption}

Table1. The values of $\Delta R / \Delta \theta$ for various energy of the incident particle and thickness of the bubble wall. We have chosen $g(x)=\Delta \theta f(x)^{2}$. The numerical values of $E^{*}$ and $a$ are given in the unit of $m_{0}$, the height of the wall.

\section{Figure Captions}

Figure1. $\Delta R / \Delta \theta$ as a function of $E^{*}$ for various $a$, in the case where $g(x)=\Delta \theta f(x)^{2}$. The numerical values of $E^{*}$ and $a$ are given in the unit of $m_{0}$, the height of the wall.

Figure2. $\Delta R / \Delta \theta$ as a function of $E^{*}$ for various $a$, in the case where $g(x)=\Delta \theta \frac{d}{d x} f(x)$. The numerical values of $E^{*}$ and $a$ are given in the unit of $m_{0}$, the height of the wall.

\begin{tabular}{|c|c|c|c|c|}
\hline$a$ & $E^{*}=1.001$ & 1.5 & 2.0 & 5.0 \\
\hline 0.1 & 0.152 & $2.32 \times 10^{-18}$ & $-2.42 \times 10^{-26}$ & $-2.42 \times 10^{-70}$ \\
\hline 0.2 & 0.387 & $9.12 \times 10^{-10}$ & $4.96 \times 10^{-14}$ & $4.69 \times 10^{-36}$ \\
\hline 0.4 & 0.277 & $5.98 \times 10^{-6}$ & $1.07 \times 10^{-8}$ & $-6.23 \times 10^{-20}$ \\
\hline 0.6 & -1.14 & $8.71 \times 10^{-6}$ & $-2.06 \times 10^{-7}$ & $-1.15 \times 10^{-13}$ \\
\hline 0.8 & -0.640 & $-1.00 \times 10^{-3}$ & $-6.50 \times 10^{-5}$ & $-1.37 \times 10^{-10}$ \\
\hline 1.0 & -0.474 & $-3.12 \times 10^{-3}$ & $-3.19 \times 10^{-4}$ & $-6.94 \times 10^{-9}$ \\
\hline 2.0 & -0.244 & $-1.61 \times 10^{-2}$ & $-4.28 \times 10^{-3}$ & $-1.14 \times 10^{-5}$ \\
\hline 4.0 & -0.189 & $-2.44 \times 10^{-2}$ & $-1.00 \times 10^{-2}$ & $-3.02 \times 10^{-4}$ \\
\hline 10.0 & -0.174 & $-2.69 \times 10^{-2}$ & $-1.29 \times 10^{-2}$ & $-1.32 \times 10^{-3}$ \\
\hline 20.0 & -0.172 & $-2.73 \times 10^{-2}$ & $-1.33 \times 10^{-2}$ & $-1.74 \times 10^{-3}$ \\
\hline 40.0 & -0.172 & $-2.74 \times 10^{-2}$ & $-1.34 \times 10^{-2}$ & $-1.87 \times 10^{-3}$ \\
\hline
\end{tabular}

Table 1 


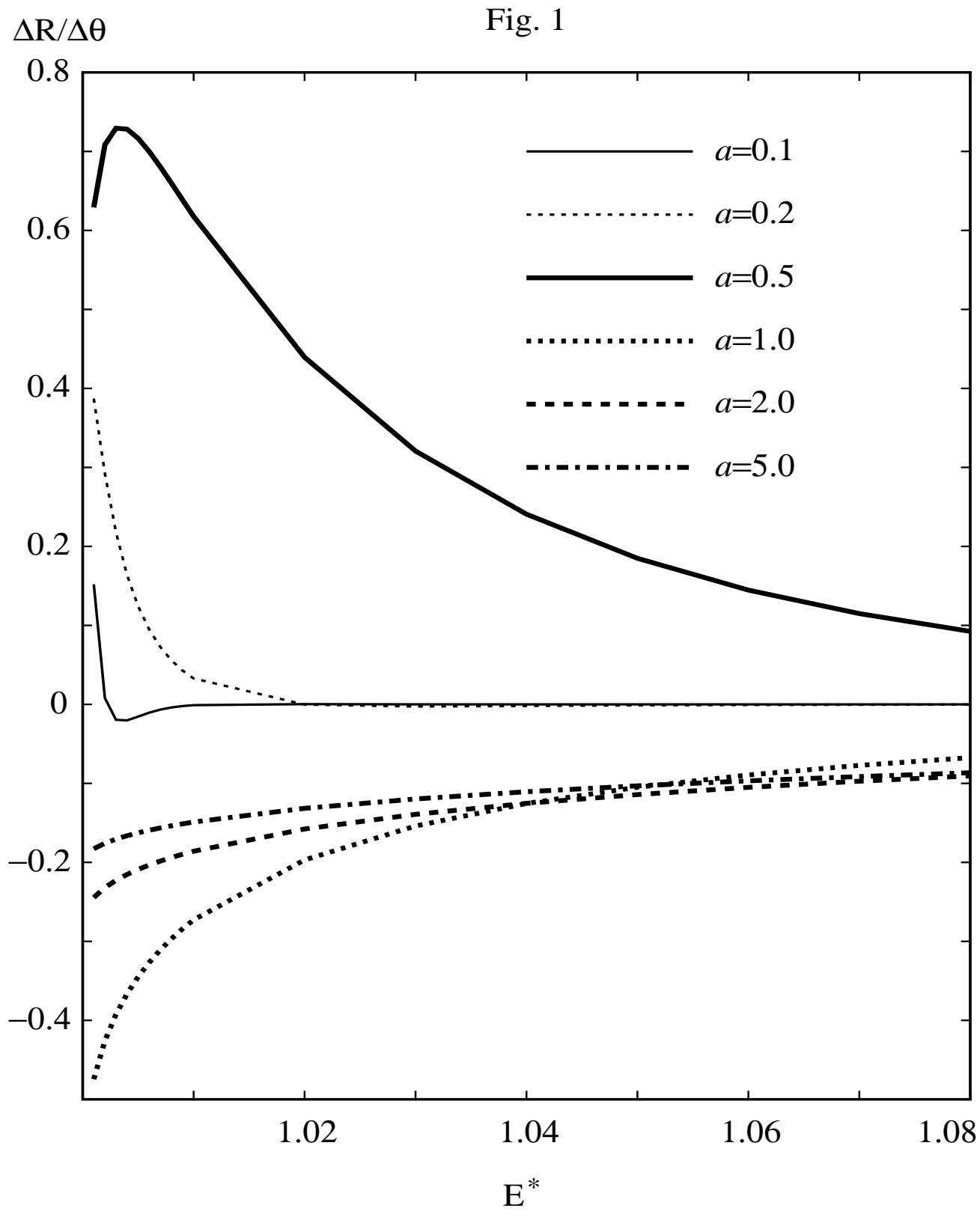




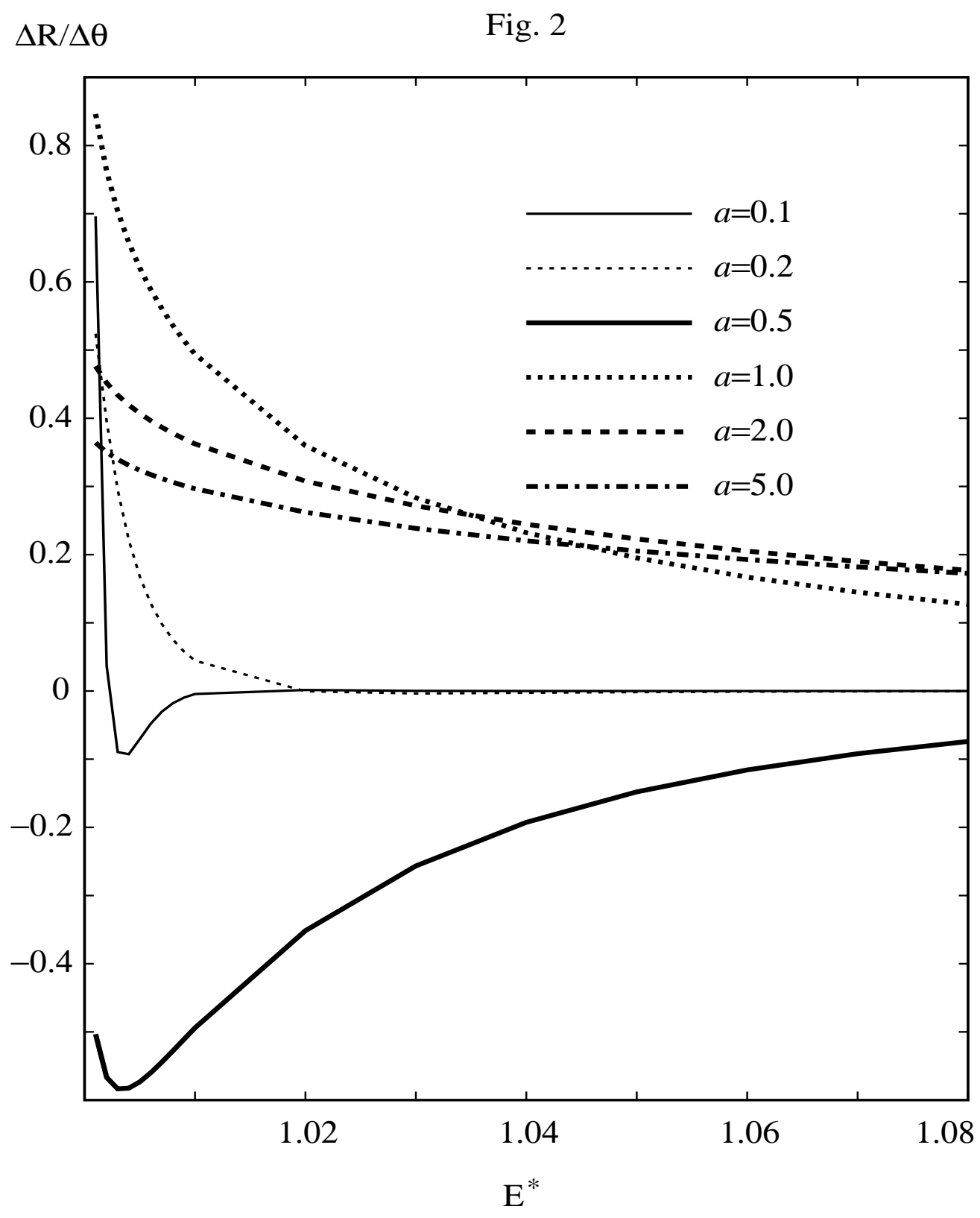

